EDUCATION AND TRAINING

\title{
Models of the medical consultation: opportunities and limitations of a game theory perspective
}

\author{
C Tarrant, T Stokes, A M Colman
}

The medical consultation is best understood as a two-way social interaction involving interactive decision making. Game theory-a theory based on assumptions of rational choice and focusing on interactive decision making - has the potential to provide models of the consultation that can be used to generate empirically testable predictions about the factors that promote quality of care. Three different game structures - the Prisoner's Dilemma game, the Assurance game, and the Centipede game-all provide insights into the possible underlying dynamics of the doctor-patient interaction. Further empirical work is needed to uncover the underlying game structures that occur most commonly in medical consultations. Game theory has the potential to provide a new conceptual and theoretical basis for future empirical work on the interaction between doctors and their patients.

See end of article for authors' affiliations

Correspondence to: Carolyn Tarrant, Division of General Practice and Primary Health Care, Department of Health Sciences, University of Leicester, Gwendolen Road, Leicester LE5 4PW, UK; ccp3@le.ac.uk

Accepted for publication 30 August 2004
$\mathrm{T}$ he medical consultation is best understood as a two-way social interaction. In a typical consultation the doctor elicits information from the patient, then offers a diagnosis or opinion and may also discuss and offer treatment. The patient can choose what information to disclose and how to present it, can ask questions that influence the doctor's perception of the problem, can make explicit requests and, above all, can choose how to respond to the advice offered or the treatment prescribed. The outcome of the consultation is affected by the actions and choices of both participants. In other words, a consultation involves interactive decision making. The closely related notion of shared decision making - the idea that doctors should collaborate with patients in making treatment or management decisions-has been the focus of extensive conceptual and empirical research, particularly in the context of primary care. ${ }^{12}$ There is little evidence that shared decision making routinely happens in consultations, ${ }^{3}$ but it is clear that even if the decision making process is not shared, the outcome of the consultation will still usually depend on the choices of both the doctor and the patient- the doctor's decision about treatment or management and the patient's decision about whether or not to follow the advice or prescribed treatment.

A conceptual apparatus for describing and analysing interactive decision making is supplied by game theory. The theory emerged in the $1940 s^{4}$ following preliminary work by the French mathematician Borel and the Hungarian mathematician von Neumann in the 1920s and 1930s. Its influence in the social and behavioural sciences began to grow after the publication of a more accessible account of the theory by Luce and Raiffa, ${ }^{5}$ culminating in the award of Nobel prizes to three leading game theorists in 1994. Game theory has found wide application in social psychology where it has been used to model decision making in a range of contexts ${ }^{6}$ including economics, ${ }^{78}$ politics, ${ }^{910}$ and biological sciences. ${ }^{11}$

Hockstra and Miller $^{12}$ were among the first to recognise the interactive nature of decision making in medical consultations, and hence the value of game theory in modelling this decision making process. There has also been some interest in the use of game theory to develop prescriptive models of medical decision making. ${ }^{13}$ Game theory has the potential, however, to provide a valuable theoretical basis for broader questions about the medical consultation. This approach has received little attention, with the exception of the work by Batifoulier which explored the relevance of game theory models to the doctor-patient interaction, and drew on this theoretical perspective to address the question of what produces cooperation between the doctor and the patient. ${ }^{14}$ Palombo also used game theory principles as the basis of a discussion on the development of the therapeutic alliance in psychiatry. ${ }^{15}$

Game theory may have particular value in increasing our understanding of doctor-patient relationships. A recent narrative review of empirical research has found evidence that continuing relationships between doctors and patients are associated with a range of measurable positive outcomes, including quality of care, adherence to treatment, and patient satisfaction, but may also be associated with negative outcomes including poorer control in diabetic patients and difficulty in the application of evidence based care. ${ }^{16}$ However, much of this research is pragmatic, lacking a theoretical basis through which findings can be integrated and from which new hypotheses can be developed and tested. The theoretical and experimental literature on game theory and experimental games includes a huge body of research on the factors promoting cooperation, reciprocity and trust, which could be applied to developing an understanding of cooperation and trust in the consultation.

Work carried out by Gutek and colleagues in the US and Australia ${ }^{17-19}$ provides a good example of the use of game theoretic principles to model the organisation of service provision and its 
impact on service quality. Based on game theoretic principles, Gutek asserted that continuing relationships between providers and consumers are conceptually distinct from the other modes of service provision and have unique features that help to promote cooperation and quality of care. Her empirical work provided evidence to support this assertion. Customers who received service within relationships were more likely to trust their providers and recommend their providers to others. They reported more personalised service within relationships and were more likely to direct complaints to their individual providers than to managers. ${ }^{19}$ Service relationships were also found to be linked to higher customer satisfaction and higher frequency of service use. ${ }^{18}$ Gutek's work provides an illustration of the use of game theory to develop a theoretical model and to generate and test predictions about service quality. Although this work did not have a specific focus on medical care, it does point to the value of further research using game theory models to identify predictors of quality in health care.

We propose that game theory has the potential to provide models of the consultation and its organisational contextmodels that can be used to generate empirically testable predictions about the factors that promote good quality health care. In this paper we (1) introduce the key concepts of game theory; (2) outline some game structures and evaluate their relevance to the medical consultation in primary care; and (3) discuss the possible contribution of game theory models to research into the consultation and quality of health care. The paper focuses particularly on doctor-patient interactions in the context of primary care. However, all medical consultations are social interactions and, as such, it is likely that this approach will have relevance to understanding medical consultations more generally.

\section{KEY CONCEPTS OF GAME THEORY}

Game theory is concerned with decisions in which the outcomes depend on the actions of two or more decision makers, called players, and where each player has two or more ways of acting, called strategies. Each player is assumed to have clear preferences among the possible outcomes. The theory is not concerned with the sources and nature of preferences and beliefs but assumes that people generally try to do the best for themselves in the light of their beliefs. ${ }^{20}$ Essentially, game theory provides a means of abstracting the fundamental structure of an interaction and representing it in terms of a strategic game.

\section{Prisoner's Dilemma game}

The most famous of all strategic games, the Prisoner's Dilemma game, is a standard model of a two-person interaction involving cooperation and competition, or trust and betrayal. Its name comes from a scenario involving two people, arrested and charged with involvement in a serious crime, held in separate cells and prevented from communicating with each other. The police have insufficient evidence for a conviction unless at least one of the prisoners discloses incriminating information. Each prisoner is faced with a choice between concealing information $(C)$ and disclosing it $(D)$. If both conceal the information, then both will be acquitted. If both disclose the information, then both will be convicted. If only one prisoner discloses the information, then that prisoner will be acquitted and will also receive a reward for helping the police (the best possible outcome), while the prisoner who conceals the information will receive an especially heavy sentence (the worst possible outcome). It is customary to interpret the $C$ strategy as cooperate and the $D$ strategy as defect.

The underlying structure of the Prisoner's Dilemma game is shown in fig 1 .

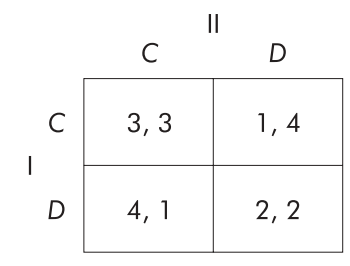

Figure 1 Prisoner's Dilemma game. Player I chooses between rows $C$ (cooperate) and D (defect), player II chooses between columns $C$ and $D$, and the numbers in each cell represent the payoffs to player I and player II in that order, 4 being best, 3 second best, 2 third best, and 1 worst.

A key concept in game theory is the Nash equilibrium, named after the Nobel laureate John Nash, the subject of a biography by Sylvia Nasar ${ }^{21}$ and an Oscar winning film entitled "A Beautiful Mind". For a two player game, a Nash equilibrium is a pair of strategies that are best replies to each other, a best reply being a strategy that yields the best outcome to the player choosing it, given the co-player's strategy. The unique Nash equilibrium in the Prisoner's Dilemma game is joint defection $(D, D)$, with both prisoners disclosing information. This is because $D$ is a best reply for both players in the sense that it yields a better outcome to each individual than cooperating, whether the co-player chooses to cooperate or to defect. Although both prisoners would be better off if they both chose to conceal the information $(C, C)$, joint cooperation is not in equilibrium. This is because the best reply to a cooperative $(C)$ strategy is defection $(D)$ - a prisoner who discloses information while the other conceals it is not only acquitted but also rewarded. And by choosing $C$, a prisoner exposes himself to the risk of the worst possible outcome for himself-the possibility of an especially heavy sentence if the other prisoner succumbs to the temptation of a reward for confessing. $(D, D)$ is the only outcome in which each player's strategy is a best reply to the co-player's. From a purely game theoretic point of view, cooperation is never a rational strategy in a one-off Prisoner's Dilemma game.

\section{Prisoner's Dilemma and the consultation}

With reasonable simplifying assumptions, medical consultations in primary care may have an underlying structure that corresponds to the Prisoner's Dilemma game. In any consultation it is possible for the doctor either to act in the patient's best interests $(C)$ or (whether through error, misjudgement, lack of skills, or conflicting goals) to take a course of action that is not in the best interests of the patient $(D)$, leading to poor quality care. The patient, in any given consultation, has to decide whether to go along with the doctor's advice or prescribed course of treatment $(C)$, or not (D).

The following hypothetical scenario will provide an intuitive interpretation of a prisoner's dilemma in a medical consultation. Let us suppose that during a busy Friday afternoon surgery at a general practice, a doctor is consulted by an adult patient who has had a sore throat for several days. The examination findings reveal a red throat, a slight fever, and slightly swollen cervical lymph nodes. The doctor considers whether, on the one hand, to give the patient a prescription for antibiotics, hence dealing with the patient in less than 5 minutes or, on the other, to undertake a full assessment of lifestyle and other contributing factors and to give tailored written advice about self-management which would prolong the consultation to over 10 minutes. The patient can choose either to follow the course of treatment/ advice or not to follow the course of treatment/advice (and to take other action which might include consulting another doctor in the same general practice for a second opinion). There are four possible outcomes:

- $(C, C)$ : the doctor spends time giving advice; the patient chooses to follow the advice. 
- $(C, D)$ : the doctor spends time giving advice; the patient chooses not to follow the advice.

- $(D, C)$ : the doctor gives a prescription; the patient follows the course of treatment.

- $(D, D)$ : the doctor gives a prescription; the patient does not follow the course of treatment.

In this example there is an intuitive sense in which $(C, C)$ is best all round-the doctor does best by the patient and the patient follows the doctor's advice without taking up valuable time of other doctors. But this outcome is not a Nash equilibrium. The unique Nash equilibrium is joint defection $(D, D)$, as explained earlier. By choosing $D$, both doctor and patient avoid the risk of the worst possible outcome for themselves-that is, cooperating when the other player chooses to defect. If the doctor chooses to deal with the patient quickly by issuing a prescription rather than spending time to find a more appropriate management option, then the best outcome for the patient is achieved by choosing not to follow through with the treatment and getting a second opinion; and if the patient decides in this way, then the best option for the doctor is to deal with the patient quickly. However, $(D, D)$ would clearly not make for good quality care and is obviously not the most desirable outcome. That is why the Prisoner's Dilemma game, and actual interactions which have its strategic structure, are considered somewhat paradoxical and problematic.

\section{Factors promoting cooperation}

In a single-play Prisoner's Dilemma game as described above, game theoretic principles show that cooperation is not a rational strategy. However, the situation is different when we consider interactions that are expected to continue indefinitely in the future. Evolutionary game theory, which rose to prominence in the 1980s, stimulated primarily by the work of Maynard Smith ${ }^{11}$ and Axelrod, ${ }^{90}$ focuses precisely on such indefinitely repeated games. Analysis of the indefinitely repeated Prisoner's Dilemma game reveals that, in this context, it is possible to find cooperative strategies that are Nash equilibria. ${ }^{22}$ This suggests that cooperation is a rational strategy only when interactions are embedded in a sequence of repeated contacts that are expected to continue indefinitely in the future. Important factors that promote cooperation include players' anticipation of future interactions and their ability to recognise each other and recall past interactions. ${ }^{9324}$ If players anticipate interacting again in the future, then they can foresee future payoffs from mutual cooperation. In addition, the threat of recrimination from the other player in future interactions acts as a disincentive to defection.

In the context of the consultation, mutual cooperation becomes a more attractive prospect if future interactions are anticipated. There are incentives for the doctor to spend time finding an appropriate management approach: consultations with the same patient in the future are likely to take up less time and the doctor will have the satisfaction of carrying a management plan through to completion. The patient is likely to follow through with the treatment if there is an expectation that the doctor will monitor his progress in the future. Both the doctor and the patient can anticipate future payoffs from this mutual cooperation, and this model implies that higher quality of care can be achieved when the patient sees the same GP repeatedly.

This consideration lies at the heart of the service quality models proposed by Gutek ${ }^{17-19}$ and provides a valuable theoretical basis for models of the organisation of health care-in particular, the role of doctor-patient relationships in providing good quality care.

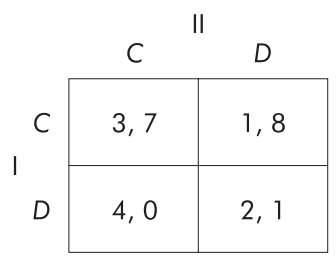

Figure 2 Modified Prisoner's Dilemma game. In this example the outcomes matter much less to the doctor (player I) than to the patient (player II)-in particular, the doctor's action has a much greater impact on the patient than vice versa.

\section{Unequal payoffs in the Prisoner's Dilemma game}

There are some potential problems with assuming that the Prisoner's Dilemma game would be an appropriate model of doctor-patient interactions. In particular, the Prisoner's Dilemma game as described above models player I and player II as interchangeable-the payoffs to each player under each condition are of the same value. However, in reality the doctor and patient occupy very different roles. In many ways the stakes are higher for patients than for doctors-it may be crucial for a patient that the doctor gives the most appropriate care or treatment, but a patient's choice of whether or not to follow advice may have little or no implication for the doctor. It may be more plausible to think of a medical consultation as having the basic strategic structure of the Prisoner's Dilemma game but with the payoffs having a far greater impact on the patient than the doctor (fig 2). As in any Prisoner's Dilemma game, each player benefits from the co-player's cooperation and both are better off if both cooperate than if both defect. But in fig 2 the payoffs reflect the fact that the doctor's (player I's) cooperation has a large impact on improving the patient's (player II's) payoffs, whereas the patient's cooperation leaves the doctor only slightly better off. Each player has reason to hope for cooperation from the other, and joint cooperation is in both players' interests, but it is more important to the patient that the doctor cooperates (by providing appropriate treatment or management advice) than it is to the doctor that the patient cooperates.

\section{OTHER GAME THEORY MODELS \\ Assurance game}

The Prisoner's Dilemma game assumes that there is a conflict between self-interest and the benefits achieved through mutual cooperation. However, it may be that some types of medical consultation are more accurately represented as coordination games ${ }^{25}$ where both the doctor and patient benefit most from joint cooperation. The Assurance game, introduced by Sen, ${ }^{26}$ models interactions where mutual cooperation is the best possible outcome, but where cooperation may involve an element of risk. In such situations both players need assurance or trust to risk cooperation. The Assurance game is shown in fig 3.

Sen gave the following illustrative interpretation of the game. Two people face the choice of going to a lecture $(C)$ or staying at home $(D)$. Both regard going to the lecture together to be the best alternative; both consider staying at home together to be the next best; and each considers going to the lecture without the other worst. The Assurance game differs from the Prisoner's Dilemma in that the $(C, C)$

\begin{tabular}{c|c|c|}
\multicolumn{4}{c}{$C$} & \multicolumn{1}{c}{$C$} & \multicolumn{1}{c}{$D$} \\
\cline { 2 - 3 } \multicolumn{1}{c|}{$C$} & 4,4 & 1,2 \\
\cline { 2 - 3 } & & 1,4 \\
\cline { 2 - 3 } & 2,1 & 3,3 \\
\hline
\end{tabular}

Figure 3 Assurance game: cooperation involves risk and therefore requires trust. 
outcome is a Nash equilibrium-neither player can do better than to cooperate if the other chooses to cooperate. For player I, $C$ is the best reply to player II's $C$, and for player II, $C$ is the best reply to player I's $C$. But there is another Nash equilibrium at $(D, D)$, where strategies are also best replies to each other. Furthermore, the Nash equilibrium that both prefer (that is, mutual cooperation) involves greater risk because each has to risk the worst possible payoff if they choose to cooperate and the co-player defects. This game models situations in which both participants are better off working together, but if either defects from a cooperative mode of interaction it is best for the other to do likewise because unilateral cooperation yields the worst possible payoff to the cooperator and does not bring much benefit to the co-player.

One intuitive example of the Assurance game in the context of a medical consultation might be health promotion activity, such as a doctor initiating a patient onto a smoking cessation programme. Clearly both the doctor and the patient are better off if the smoking cessation programme is initiated, but without the assurance that the patient will cooperate the doctor risks substantial losses. The worst payoff for the doctor would come from putting time and effort into initiating a programme when the patient has no intention of cooperating. Without assurance, the best strategy for the doctor would be defection-that is, not attempting to initiate the programme at all as this minimises the risk of ending up with the worst payoff.

Trust and assurance are crucial in promoting cooperation in the Assurance game. If each player can be relatively confident that the other will cooperate, then mutual cooperation is a likely outcome. As in the case of the Prisoner's Dilemma game, the outcome will be influenced by whether the interaction is a "one-off" or is in the context of a series of repeated interactions. If the doctor and patient have a history of past interactions, then each will have information about the other on which to base judgements of how likely the other is to cooperate. Also, if the doctor and patient anticipate interacting again in the future, each can make clear their commitment to working together. Communication in the consultation is also likely to play an important role in the assessment of trust and assurance. The Assurance game may provide a valuable basis for research into trust in the consultation.

\section{Centipede game}

Both the Prisoner's Dilemma game and the Assurance game provide models of single interactions, although it is possible for these games to be played repeatedly by the same two players, each time with the same basic game structure. The two-person Centipede game, introduced by Rosenthal, ${ }^{27}$ is less well known but is attracting increasing attention from game theorists. Rather than providing a complete model of a single interaction, the Centipede game is designed to model interactions repeated a certain number of times between a pair of players. A simple version with just two moves for each

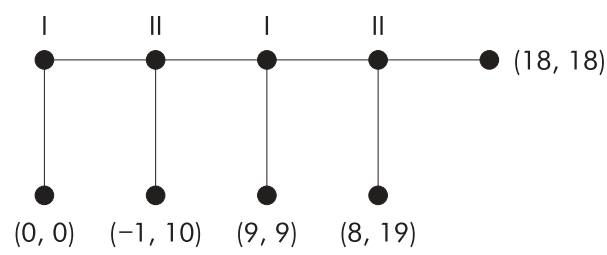

Figure 4 Centipede game. Starting at the left, players I and II alternate in choosing whether to defect by moving down or to cooperate by moving across. If a player defects, then the game stops at that point and the payoffs are shown in parentheses (in the order I, II). player each is shown in fig 4, but the game could be extended to any number of moves.

The sequence of moves starts at the left. Players I and II alternate in choosing whether to defect (by moving down) or to cooperate (by moving across). If either player defects at any point, then the game stops and the players receive the payoffs shown in parentheses (player I's on the left, as usual). For example, if player I defects down on the first move, then the game stops and both payoffs are zero. But whenever a player makes a cooperative (across) move, that player loses 1 unit and the co-player gains 10. If player I cooperates on the first move, losing 1 unit and adding 10 to player II's payoff, and if player II promptly defects down, then the game stops and the payoffs are -1 to player I and 10 to player II, and so on. If both players cooperate on every move, then the game ends automatically after the fourth decision with a payoff of 18 to each player. The game could, of course, be much longer with even larger payoffs towards the Centipede's head.

This game has much in common with the Prisoner's Dilemma game, because both games model two-person interactions in which joint cooperation is in the interests of both players but each faces a temptation (in Centipede, an escalating sequence of temptations) to defect. In both games the players benefit from each other's cooperation. But the Centipede game obviously models a continuing relationship in which the mutual benefit of cooperation increases over successive encounters, and in which mutual trust and trustworthiness are essential if cooperation is to be possible. This is often seen in the context of the doctor-patient relationship. Investment over time by the doctor and patient in an ongoing relationship can bring increasing mutual benefit-the doctor's personal knowledge of the patient is often helpful in making appropriate diagnosis and management plans, and the patient may have increasing confidence in the treatment. However, this is difficult if the doctor or patient are unable to rely on the other's cooperation.

The Centipede game provides an illuminating model of dyadic relationships in which the temptation to defect at each stage threatens the sequence of increasing payoffs. Patients and doctors may choose to defect from the relationship in a number of different ways. A defecting move by a patient might be non-acceptance of treatment or advice or, at the extreme, may involve literally terminating the relationship by switching to a different doctor. Defection by the doctor may involve asking the patient to see a different doctor, or stopping the Centipede game without actually breaking off the relationship. The latter might occur, for example, in response to a perceived abuse of the doctor-patient relationship by the patient, following which the doctor and patient continue to see each other in medical consultations but the relationship of trust is severed and the increasing mutual gains from reciprocal cooperation no longer apply. The Centipede game may also act as a useful model of interactions within a single consultation where a sequence of cooperative moves by a patient and doctor (for example, expression of empathy by doctor, disclosure of additional symptoms by patient) may build to a mutually rewarding outcome. In contrast, defection at any stage (for example, a rejection by the patient of the doctor's suggested diagnosis) may lead to termination of the consultation without benefit to either party.

Rosenthal $^{27}$ showed that defection is always rational, and the only Nash equilibrium involves player I defecting on the very first move. To see why this is so, consider the last decision node where player II can either defect and gain 19 or cooperate and gain 18. A rational player II would defect, but that means that, on the previous move, player I would anticipate this and would defect to gain 9 rather than 8 , and 
this argument can be extended back to the first move. This conclusion highlights a weakness of formal game theory because intuition suggests, and experiments have confirmed, that people are far more cooperative-almost all cooperating on the first move and many even on the last—and as a result they gain substantial benefits. This is a striking example of human reciprocal cooperation with mutual benefit. Behavioural game theory, which has recently begun to attract a great deal of interest across the social and behavioural sciences, ${ }^{7}$ is devoted precisely to discovering and explaining behaviour of this kind, even (or especially) when it deviates from the formal prescriptions of orthodox game theory.

\section{DISCUSSION}

This review has indicated that game theory can be applied to the medical consultation and used to generate predictions about how the context of a doctor-patient interaction influences cooperation and quality of care. In particular, game theory models indicate that a history of past interactions between a doctor and patient and anticipation of future interactions make cooperation and good quality care more likely.

These principles formed the basis of the work by Gutek ${ }^{17}$ who inferred that ongoing provider-customer relationships promote mutual cooperation and improved quality of service. Gutek's work highlights the potential for the use of game theory in the organisation and provision of health care, but this needs to be developed further. Not all repeated contacts between doctors and patients lead to cooperation; for example, some ongoing relationships are problematic and, in some cases, cooperation is difficult (or impossible) to get going. So-called "heartsink" patients are a clear example of this. ${ }^{28}$ There would be value in further research based on predictions drawn from game theory to investigate which features of repeated doctor-patient interactions are more or less likely to lead to mutual cooperation and good quality care.

The Prisoner's Dilemma is the most commonly used game structure in game theoretic research. ${ }^{29}$ However, there are many other possible game structures that might be appropriate models of the medical consultation, including the Assurance and Centipede games. We have given intuitive examples of how game theory models might apply to the medical consultation, but empirical work to identify and develop appropriate game theoretic models of the medical consultation would be of great value, and would open up further possibilities for the use of applied game theory in consultation research.

The development of game theory models of the doctorpatient interaction presents an interesting challenge because of the different and non-interchangeable roles of the doctor

\section{Key messages}

- Game theory provides a new perspective for research into the medical consultation.

- Doctors and patients have identifiable goals and aspirations, and this makes game theoretic models potentially relevant.

- Different game structures give insight into the underlying dynamics of different types or aspects of doctorpatient interactions.

- Game theory can provide the basis for empirically testable models of the doctor-patient interaction and the factors that promote quality of care. and patient within the interaction. Game theory models generally assume that the order of preference for outcomes is identical for both players, and that the players are essentially interchangeable. However, the doctor and patient may differ greatly in the value they put on different consultation outcomes, and their preferences for different outcomes may not be symmetrical. This needs to be addressed in future research.

Game theory allows us to model, and to make predictions about, the impact on trust, cooperation, and quality of care of contextual factors such as whether participants anticipate interacting in the future, whether they have a history of past interaction, and so on. These factors are potentially important, over and above the familiar and well researched individual factors such as the interpersonal or communication skills of the participants. Furthermore, contextual factors are potentially important inasmuch as the organisation of care is more amenable to change than are ingrained individual characteristics. Insight into the effects of contextual factors on cooperative interaction in primary health care also opens up the possibility of predicting how organisational changes are likely to impact on these important process and outcome variables. Game theory provides a suitable conceptual framework within which past findings can be brought together into an integrated model to generate new hypotheses which can then be tested empirically.

Game theory was originally devised to model purely rational decision making in strategic interactions. Decades of experimental research have revealed, however, that human decision makers deviate from the prescriptions of the theory in certain games, including the Prisoner's Dilemma and Centipede games. ${ }^{20}$ In particular, human decision makers often behave far more cooperatively than the theory predicts. Psychological game theory ${ }^{6}$ and behavioural game theory ${ }^{7}$ have been developed specifically to explain such behaviour and to provide more accurate models of real human interaction. This new and developing field has much to contribute to new understandings of the doctorpatient interaction and its organisational context.

\section{CONCLUSIONS}

Game theory can allow us to represent some of the fundamental features of medical consultations and their organisational context, and the theory provides a strong conceptual and theoretical basis for empirical work from which it is possible to generate empirically testable hypotheses about interactions between patients and doctors. While this review has illustrated the applicability of game theory to models of the medical consultation, it is acknowledged that game theory has a wider applicability to different healthcare provider-patient interactions. The authors are currently involved in a programme of work to develop the application of game theory to the medical consultation in primary care.

\section{ACKNOWLEDGEMENTS}

The authors thank Kate Windridge for reviewing a draft of this paper; Glyn Elwyn, Fiona Moss and the reviewers for their constructive feedback; and Andrew Sant, University of Leicester Royal Literary Fund Writing Fellow for advice. The contribution of the third author was supported in part by grant no. RES-000-23-0154 from the Economic and Social Research Council.

\section{Authors' affiliations}

C Tarrant, T Stokes, Division of General Practice and Primary Health Care, Department of Health Sciences, University of Leicester, Leicester General Hospital, Leicester LE5 4PW, UK

A M Colman, School of Psychology, University of Leicester, Leicester LE 1 $7 \mathrm{RH}, \mathrm{UK}$ 


\section{REFERENCES}

1 Charles C, Gafini A, Whelan T. Shared decision-making in the medical encounter: what does it mean (or it takes at least two to tango). Soc Sci Med 1997;44:681-92.

2 Elwyn G, Edwards A, Kinnersley P. Shared decision making in primary care: the neglected second half of the consultation. Br J Gen Pract 1999:49:477-82.

3 Stevenson FA, Barry CA, Britten N, et al. Doctor-patient communication about drugs: the evidence for shared decision-making. Soc Sci Med 2000;50:829-40.

4 von Neumann J, Morgenstern O. Theory of games and economic behavior. Princeton, NJ: Princeton University Press, 1944

5 Luce RD, Raiffa H. Games and decisions: introduction and critical survey. New York: Wiley, 1957.

6 Colman AM. Cooperation, psychological game theory, and limitations of rationality in social interaction. Behav Brain Sci 2003;26:139-53.

7 Camerer CF. Behavioral game theory: experiments in strategic interaction. Princeton, NJ: Princeton University Press, 2003.

8 Sugden R. Rational choice: a survey of contributions from economics and philosophy. Economic J 1991;101:751-85.

9 Axelrod R. The evolution of cooperation. New York: Basic Books, 1984.

10 Axelrod R. The complexity of cooperation: agent-based models of competition and collaboration. Princeton, NJ: Princeton University Press, 1997.

11 Maynard Smith J. Evolution and the theory of games. Cambridge, UK: Cambridge University Press, 1982.

12 Hockstra DJ, Miller SD. Sequential games and medical diagnosis. Computers Biomed Res 1976;9:205-15.

13 Diamond GA, Rozanski A, Stever M. Playing doctor: application of game theory to medical decision-making. J Chronic Dis 1986;39:669-82.

14 Batifoulier P. L'Interaction medicin-patient: un examen par la theorie des jeux. Cah Socio Demo Med 1997;37:341-64.
15 Palombo SR. The Prisoner's Dilemma: game theory and the therapeutic alliance. Psychoanal Q 1997;66:628-41.

16 Pereira Gray D, Evans P, Sweeney K, et al. Towards a theory of continuity of care. J R Soc Med 2003;96:160-6.

17 Gutek BA. The dynamics of service: reflections on the changing nature of customer/provider interactions. San Francisco: Jossey-Bass, 1995.

18 Gutek BA, Bhappu AD, Liao-Troth MA, et al. Distinguishing between service relationships and encounters. J Appl Psychol 1999;84:218-33.

19 Gutek BA, Cherry B, Bhappu AD, et al. Features of service relationships and encounters. Work and Occupations 2000;27:319-52.

20 Colman AM. Game theory and its applications in the social and biological sciences, 2nd ed. London: Routledge, 1995

21 Nasar S. A beautiful mind. London: Faber \& Faber, 1998.

22 Binmore K. Fun and games: a text on game theory. Lexington, MA: Heath, 1992.

23 Murnighan JK, Roth A. Expecting continued play in Prisoner's Dilemma games. J Conflict Resolution 1983;27:279-300.

24 Fehr E, Gächter S. Altruistic punishment in humans. Nature 2002:415:137-40

25 Mehta J, Starmer C, Sugden R. Focal points in pure coordination games: an experimental investigation. Theory and Decision 1994;36:163-85.

26 Sen AK. A game-theoretic analysis of theories of collectivism in allocation. In: Majumdar T, ed. Growth and choice: essays in honour of U N Ghosal. Calcutta: Oxford University Press, 1969:1-17.

27 Rosenthal RW. Games of perfect information, predatory pricing and the chain-store paradox. J Econ Theory 1981;25:92-100.

28 O'Dowd TC. Five years of heartsink patients in general practice. BMJ 1988;297:528-30

29 Grzelak J. Conflict and cooperation. In: Hewstone M, Stroebe W, Codol J-P et al. Introduction to social psychology: a European perspective. Oxford: Basil Blackwell, 1988:288-312.

\section{Admissions for ADRs are as high as ever}

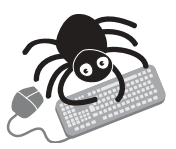

Please visit the Quality and Safety in Health Care website [www. qshc.com] for a link to the full text of this article.
$T$ he largest prospective study of adverse drug reactions (ADRs) needing hospital admission in the UK has exposed a huge burden on patients and costs to the National Health Service (NHS) and an urgent need to reduce them. Moreover, most of the reactions were predictable and preventable.

Over six months one in 16 of 18820 patients were admitted to two NHS hospitals in Merseyside, north west England, because of ADRs, $80 \%$ as a direct result and in $2 \%$ with fatal consequences. Median patient age was 76 years and median hospital stay eight days, using an estimated $4 \%$ of hospital bed capacity and translating to an estimated annual cost of up to $£ 466 \mathrm{~m}(€ 706 \mathrm{~m} / \$ 847 \mathrm{~m})$ over the entire NHS.

Almost three quarters of the ADRs were classified as avoidable, one in six was a drug interaction, and many occurred in elderly patients. Most commonly cited were low dose aspirin (with or without other drugs), diuretics, warfarin, and non-steroidal antiinflammatory drugs other than aspirin.

Prescribers must bear the responsibility for reducing ADRs, say the researchers, but other useful measures would be implementing recommendations in the National Service Framework for the Elderly and increasing pharmacists' input in prescribing.

The observational study took place between November 2001 and April 2002 in a teaching hospital and a district general hospital serving 300000 and 330 000, respectively. It included every patient aged $\geqslant 16$ years admitted over that time who was judged by careful scrutiny to have an ADR but excluded overdoses.

Previous similar studies are too old, too small, or too specialised to be useful.

A Pirmohamed M, et al. BMJ 2004;329:15-19 